\title{
Prenatal testing for late-onset neurogenetic diseases
}

\author{
Edited by G Evers-Kiebooms, MW Zoeteweij and PS Harper. 219pp. BIOS Scientific Publishers Ltd, Oxford, 2002, \\ euro 153, ISBN 1859960197
}

\author{
European Journal of Human Genetics (2003) 11, 975 . doi:10.1038/sj.ejhg.5201071
}

Predictive and prenatal testing for Huntington's disease (HD) has been possible now for more than 15 years. Several studies have been carried out to look at the way carriers and noncarriers handle their situation. Studies on prenatal testing for this disease are however limited. This book gives a good overview of what is known today.

A large part of this book is the result of a European collaborative, multidisciplinary study (BIOMED working group) on the psychosocial, ethical and legal complexity of prenatal testing for HD.

The uptake for prenatal testing has been low. From 1993 until 1998, a total of 305 tests have been performed. Most of the tests were done in the UK and the Netherlands, whereas the number of tests in France and Greece was low. However, the low uptake was not compared to the number of at-risk individuals in a particular country.

The reproductive history after predictive testing for HD was also evaluated. The number of pregnancies in the group of carriers was half the number of pregnancies in the noncarrier group. In the pregnancies of the carrier group a prenatal test was done in 2/3. Those interested in knowing data on predictive and prenatal testing in Greece-, Denmarkand the German-speaking countries Austria, Switzerland and Germany can find a lot of information about the situation until 1999. Remarkably, Greek neurologists consider predictive or prenatal testing for Huntington unjustified.

The legal protection of the unborn child and the legal aspects of termination of pregnancy (TOP) in international documents and the corresponding jurisprudence are discussed. For those who wish to know the legislation for TOP in 13 European countries a concise overview is given. Difficult questions such as does a couple have a right to know that their unborn child will have a late-onset condition for which treatment may become available in the coming years are discussed. Or does a couple have an obligation to request a TOP in light of the growing wrongful life claims?

At the time of writing this book, preimplantation diagnosis (PGD) for HD was possible in Brussels, Maastricht, London and Fairfax in the USA. Most centers only perform PGD based on direct testing where the genetic status of the future parent is known. PGD for 50\% risk individuals is possible, but there are several problems with exclusion testing of the embryo or by direct testing of the embryo following nondisclosure to the parents. These dilemmas are well discussed. Guido de Wert discusses elaborately all the ethical aspects of prenatal and preimplantation diagnosis mainly for HD.

The Lay's perspective is well illustrated by Sue Watkin and Kees Varkevisser. These authors argue that everyone should have the right to decide whether or not to raise a family. People at risk of hereditary disease should not be considered as a burden to society. Nowadays at-risk individuals have options such as predictive, and prenatal testing as well as preimplantation diagnosis. It depends however where you live if you really have access to these tests.

Alexandra Dür and Josué Feingold describe the French experience with predictive testing for autosomal dominant cerebellar ataxia.

David Craufurd describes that the uptake of prenatal testing for HD is low. Worldwide between 1986 and 1994 fewer than 250 prenatal tests were performed, in contrast to several thousands predictive tests that were performed in this same period. The possible reasons for this low uptake are discussed. A complex issue arises when a family has children at risk, and for subsequent children prenatal testing is done. When several pregnancies have been terminated because of a high risk, the emotional trauma is hard to bear, but the alternative of going ahead with an untested, at-risk pregnancy may be ineligible because a moral obligation is felt toward the earlier children. It is important for counselors to discuss the implications of the initial decision to undergo prenatal testing because these may go well beyond the existing pregnancy.

Subsequently, Martha Nance gives a review of the situation of predictive and prenatal testing for HD in the USA.

I encourage all those persons who are involved with prenatal and predictive testing for HD and other late-onset diseases to read this book. It portrays all the relevant medical, moral and ethical issues, the current laboratory techniques and the difficult choices surrounding this complex topic.

ATJM Helderman-van den Enden Department of Clinical Genetics, Section Genetic Counseling, Center for Human and Clinical Genetics, Leiden University Medical Center, Leiden, The Netherlands 